\title{
Diseminación biliar de las metástasis hepáticas del cáncer colorrectal: caso clínico y revisión de la literatura
}

\author{
Biliary growth of colorectal liver metastasis: case report and review of the literature
}

\author{
Estefanía Casas-Sicilia ${ }^{*}$, Pilar Palacios-Gasos ${ }^{1}$ Alfredo Jiménez-Bernadó1, Yaiza Martínez-Lahoz¹, Ariel \\ Gonzales-Sejas², Vicente M. Borrego-Estella y Eloy Tejero-Cebrián ${ }^{1}$ \\ ${ }^{1}$ Departamento de Cirugía General y del Aparato Digestivo; ${ }^{2}$ Departamento de Anatomía Patológica. Hospital Clínico Universitario Lozano Blesa, \\ Zaragoza, España
}

\begin{abstract}
Resumen
Introducción: El crecimiento biliar de las metástasis hepáticas del cáncer colorrectal (CCR) puede influir en su diseminación y recidiva. Caso clínico: Mujer de 65 años con antecedente de CCR (2011) y hepatectomía izquierda (2014) por metástasis hepática con afectación de la vía biliar. En 2020 desarrolló una lesión de $2 \mathrm{~cm}$ en la unión de los conductos hepáticos. Sospechando un colangiocarcinoma, se resecaron la vía biliar principal y el lóbulo caudado, asociando linfadenectomía. El estudio inmunohistoquímico confirmó el origen intestinal. Conclusiones: Una imagen de dilatación o crecimiento biliar en pacientes con antecedente de CCR debe orientar a una recidiva, aunque la imagen sugiera colangiocarcinoma.
\end{abstract}

Palabras clave: Metástasis hepáticas. Crecimiento biliar. Colangiocarcinoma. Cáncer colorrectal.

\begin{abstract}
Introduction: Intrabiliary growth of colorectal liver metastasis determines their capability of dissemination and relapse. Case report: 65-year-old woman underwent sigmoidectomy (2011) and left hepatectomy due to liver metastases affecting the bile tract (2014). In 2020 she developed a $2 \mathrm{~cm}$ tumour in the union of both hepatic ducts. Cholangiocarcinoma was suspected so resection of the main bile duct, the caudate lobe and lymphadenectomy were performed. On immunohistochemical examination, colorectal liver metastasis was confirmed. Conclusions: Liver metastases should be considered as the most likely diagnosis when bile duct dilatation or intrabiliary growth is seen in patients with a history of colorectal cancer.
\end{abstract}

Key words: Liver metastasis. Biliary growth. Cholangiocarcinoma. Colorectal cancer.

\section{Introducción}

El cáncer colorrectal (CCR) representa aproximadamente el $10 \%$ de todos los cánceres en el mundial, siendo en 2012 en Europa el tercer cáncer con mayor incidencia, por detrás de los de mama y de próstata.
En España es el cáncer con mayor incidencia si se tienen en cuenta ambos sexos'.

Las metástasis más frecuentes del cáncer de colon son las hepáticas, que aparecen aproximadamente en el $50 \%$ de los pacientes, siendo sincrónicas en el $15-25 \%^{2}$.

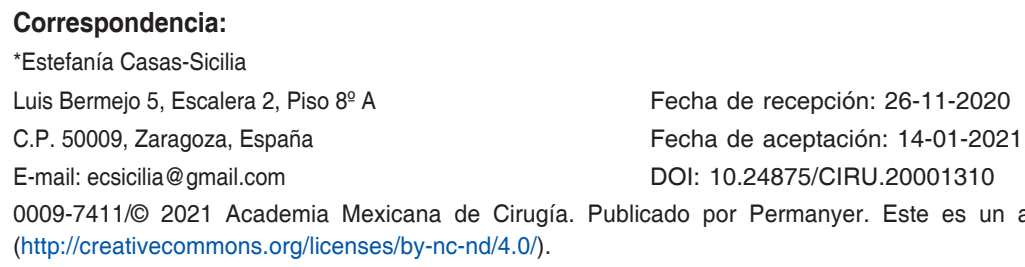


La resección quirúrgica completa es el tratamiento de elección, ya que es la única opción de tratamiento que proporciona una supervivencia aceptable a largo plazo $^{3}$. Las recidivas hepáticas ocurren en el $25-43 \%$ de los pacientes, y en muchos casos la recidiva ocurre en el margen de resección, posiblemente por la presencia de márgenes afectos en la cirugía inicial ${ }^{4}$. La supervivencia a 5 años de los pacientes intervenidos varía entre el $40 \%$ y el $67 \%$, según las series ${ }^{2,3}$.

Se ha observado que estas metástasis pueden presentar un patrón de crecimiento biliar que influye en su capacidad de diseminación y también condiciona una mayor probabilidad de recidiva local, aunque existe controversia sobre su implicación en el pronóstico a largo plazo, pues en la literatura existen resultados contradictorios.

Se presenta el caso de una paciente con metástasis hepáticas de CCR con patrón de crecimiento biliar que, tras la realización de una hepatectomía izquierda reglada, condicionó una recidiva en la vía biliar extrahepática y se planteó el diagnóstico diferencial con un colangiocarcinoma.

\section{Caso clínico}

Mujer de 65 años, hipertensa y diabética, que en 2011 fue diagnosticada de cáncer de colon descendente y fue tratada mediante colectomía izquierda por vía laparoscópica. Los valores del antígeno carcinoembrionario (CEA) en el momento del diagnóstico eran de $4.3 \mathrm{ng} / \mathrm{ml}$, y el CA 19.9 de $0.6 \mathrm{U} / \mathrm{ml}$. El examen histológico reveló que se trataba de un adenocarcinoma clásico de grado 2, sin afectación ganglionar (0/19), por lo que se clasificó como pT3NOMO. Al tratarse de un tumor estenosante y con un avance tumoral (tumor budding) de tipo infiltrante, lo cual es un factor de mal pronóstico histológico ${ }^{5,6}$, se decidió administrar quimioterapia adyuvante con capecitabina, aunque la paciente no completó el tratamiento debido al desarrollo de toxicidad en forma de diarrea y neutropenia de grado 4 que requirió ingreso hospitalario.

Durante el seguimiento, en 2014, se observó un aumento de los niveles de CEA hasta $16.23 \mathrm{ng} / \mathrm{ml}$, por lo que se solicitó una tomografía por emisión de positrones que reveló la presencia de dos metástasis en el lóbulo hepático izquierdo. La paciente fue intervenida llevando a cabo una hepatectomía izquierda reglada con colecistectomía. Se realizó una ecografía intraoperatoria que no mostró otras metástasis hepáticas. El posoperatorio evolucionó de forma satisfactoria, siendo dada de alta el séptimo día tras la intervención. En el estudio histológico de la pieza se puso de manifiesto la presencia de dos metástasis hepáticas, cada una de un tamaño aproximado de $2 \mathrm{~cm}$, de bajo grado histológico, con unos márgenes de resección libres a $>0.6 \mathrm{~cm}$, pero con una extensa invasión de la vía biliar, así como una mutación del codón 146 de K-RAS.

Tras la intervención en 2014, la paciente permaneció asintomática y sin evidencia de recidiva en los controles oncológicos practicados hasta que, en 2020, consultó en el departamento de urgencias por presentar ictericia indolora, coluria y acolia. En la analítica destacaban bilirrubina total $2.75 \mathrm{mg} / \mathrm{dl}$, bilirrubina directa $2.01 \mathrm{mg} / \mathrm{dl}$, aspartato aminotransferasa $406 \mathrm{U} / \mathrm{l}$, alanina aminotransferasa $555 \mathrm{U} / \mathrm{l}$, gamma glutamil transpeptidasa 10,999 U/l, fosfatasa alcalina $163 \mathrm{U} / \mathrm{l}$, CEA 50,3 ng/ml y CA $19.92 .48 \mathrm{U} / \mathrm{ml}$. En la tomografía computarizada (Fig. 1) se puso de manifiesto una lesión extrínseca de $23 \times 15 \mathrm{~mm}$ en la unión de las vías biliares principales junto con dilatación de la vía biliar derecha. En la colangiopancreatografía retrógrada endoscópica (CPRE) se observó una estenosis larga que afectaba al conducto hepático derecho con dilatación de las vías biliares principales proximales a la estenosis (Fig. 2), por lo que se decidió colocar una prótesis plástica. La paciente presentó, como complicación, una pancreatitis pos-CPRE que evolucionó de forma favorable con tratamiento conservador.

La paciente fue intervenida con la sospecha diagnóstica de colangiocarcinoma. Se observó una tumoración de $3 \mathrm{~cm}$ que afectaba principalmente al conducto hepático derecho, y se realizó una resección de la vía biliar principal hasta el colédoco distal, junto con resección del lóbulo caudado y una linfadenectomía hiliar, con reconstrucción mediante hepaticoyeyunostomía en Y de Roux. Intraoperatoriamente se enviaron biopsias tanto de un rodete del conducto colédoco distal como del conducto hepático derecho proximal a la resección, que resultaron negativas para malignidad.

El resultado histológico de la vía biliar principal reveló que la lesión se trataba de una metástasis de adenocarcinoma de colon, de bajo grado histológico, pero con invasión linfática y perineural. En el lóbulo caudado se observó una infiltración de los conductos biliares por adenocarcinoma de colon, así como afectación del parénquima hepático, aunque macroscópicamente no se había observado ninguna lesión. 


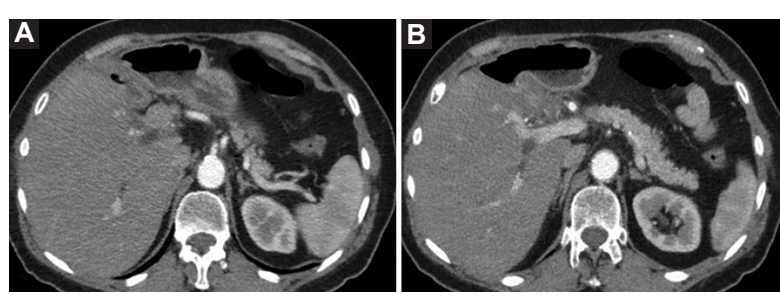

Figura 1. Tomografía computarizada que muestra una lesión de $2 \mathrm{~cm}$ en la vía biliar (A) y dilatación de vía biliar intrahepática derecha (B).

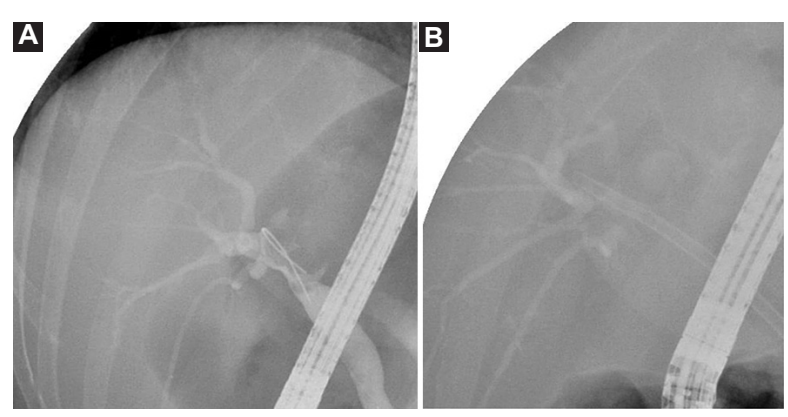

Figura 2. Colangiopancreatografía retrógrada endoscópica que muestra estenosis larga del conducto hepático derecho con dilatación de las vías biliares principales proximales (A) y colocación de una prótesis plástica (B).

Actualmente, tras 6 meses de seguimiento, no se ha evidenciado recidiva de la enfermedad.

\section{Discusión}

El crecimiento biliar de las metástasis hepáticas del CCR es un aspecto poco conocido en la actualidad, siendo la mayoría de las publicaciones estudios con un pequeño tamaño muestral. Estrella, et al. ${ }^{7}$ han reportado una prevalencia del $3.6-10.6 \%$, pero hay estudios japoneses, como el de Kubo, et al. ${ }^{8}$, que encuentra una prevalencia del $40.6 \%$, o el de Okano, et al. ${ }^{9}$, que reporta un $42 \%$, aunque solo el $12 \%$ de ellas son macroscópicas.

Se han observado dos patrones distintos de crecimiento tumoral: mediante sustitución del epitelio biliar con crecimiento a lo largo de la membrana basal, o creando «masas» hacia la luz biliar, simulando en este último caso un colangiocarcinoma ${ }^{7}$.

Por otro lado, la afectación biliar puede ser central, afectando a la vía biliar extrahepática o a los conductos biliares de primer orden, como en nuestra paciente, o periférica, afectando a los conductos biliares más pequeños. En ocasiones, la afectación de la vía biliar extrahepática es consecuencia de una metástasis parenquimatosa pequeña próxima al hilio hepático ${ }^{10}$. En nuestro caso, aunque no se había evidenciado ninguna lesión en el parénquima hepático, en los estudios radiológicos ni en el intraoperatorio, el resultado histológico definitivo sí puso de manifiesto la afectación parenquimatosa del lóbulo caudado.

El hecho de que se afectase la vía biliar extrahepática y el largo periodo libre de enfermedad (6 años desde la hepatectomía y 9 años desde la colectomía) dificultaron el diagnóstico, orientándolo inicialmente hacia un colangiocarcinoma. Además, la presentación del cuadro fue una ictericia indolora acompañada de coluria y acolia, con elevación de las enzimas de colestasis, lo que en pacientes con antecedentes de CCR puede deberse también a una masiva afectación hepática o a una recidiva adenopática que comprime la vía biliar extrahepática ${ }^{11}$. No obstante, según Coppola, et al.12, una imagen radiológica de dilatación 0 crecimiento biliar en un paciente con antecedente de CCR debe orientar a una recidiva aunque las imágenes puedan sugerir un colangiocarcinoma.

Los hallazgos radiológicos de las metástasis hepáticas de CCR con afectación biliar son muy variables y dependen principalmente de si la afectación es central o periférica ${ }^{10}$. En caso de afectación central, es decir, de la vía extrahepática o de los conductos biliares de primer orden, puede deberse a una metástasis parenquimatosa contigua, aunque en ocasiones la afectación parenquimatosa no es obvia en las pruebas de imagen y esto puede confundir el diagnóstico con un colangiocarcinoma. En la afectación periférica, en la mayoría de los casos se observará una metástasis parenquimatosa que asocie dilatación de uno o más conductos biliares, aunque en otras ocasiones la afectación parenquimatosa es mínima y puede pasar desapercibida. También pueden simular un pequeño trombo en una rama portal, así como verse áreas hipervascularizadas como consecuencia de un aumento del flujo arterial. En otros casos pueden aparecer calcificaciones lineales siguiendo un trayecto biliar $^{10}$.

Como se acaba de comentar, a veces el diagnóstico diferencial entre metástasis hepáticas de CCR y colangiocarcinoma no está claro antes de la cirugía. Sin embargo, la biopsia percutánea no se recomienda porque se asocia a diseminación tumoral extrahepática que sí repercute sobre la supervivencia global ${ }^{12} y$, en cualquier caso, no cambia la indicación ni la estrategia quirúrgica, con excepción de 


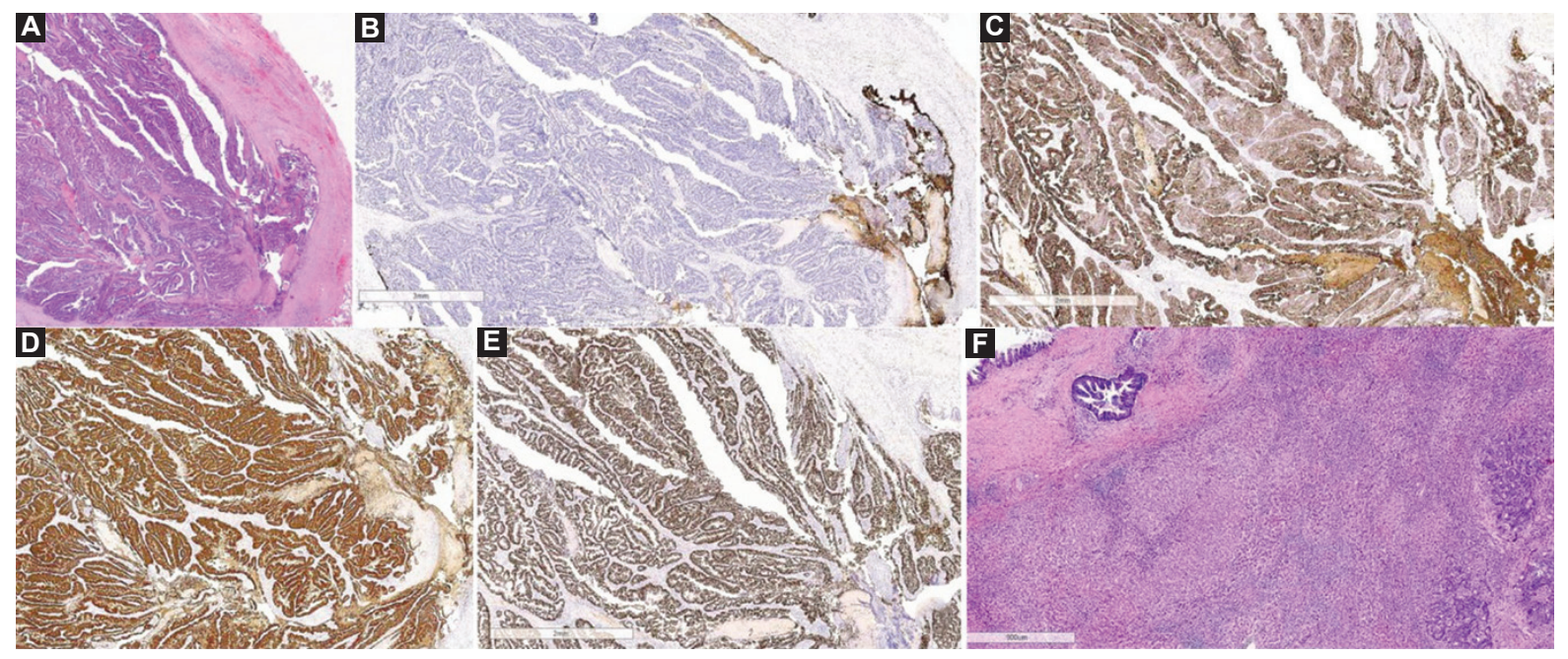

Figura 3. Estudio inmunohistoquímico. A: tinción de hematoxilina-eosina. Crecimiento endoluminal de adenocarcinoma colónico en la vía biliar, con invasión de la pared. B: negatividad para CK7, con control interno del epitelio conservado de la vía biliar. C: positividad para CK20 en la tumoración. D: intensa positividad para CDX2 en el tumor. E: intensa positividad en el tumor para SATB2. F: tinción de hematoxilina-eosina. Infiltración hepática (derecha) y afectación del conducto biliar (izquierda).

la linfadenectomía, cuyo papel sí es importante en el tratamiento de los colangiocarcinomas, pero no está claro en el caso de las metástasis hepáticas de $\mathrm{CCR}^{13}$. En ocasiones, la confirmación diagnóstica que diferencia entre metástasis hepáticas de CCR y colangiocarcinoma la aporta el estudio inmunohistoquímico ${ }^{7}$.

La distinción anatomopatológica entre colangiocarcinoma y metástasis con invasión biliar es difícil si solo se utiliza hematoxilina-eosina, siendo de gran utilidad las técnicas de inmunohistoquímica. La mayoría de las metástasis de cáncer de colon son CK7y $\mathrm{CK} 20+$, mientras que los tumores originados en el epitelio de la vía biliar suelen ser CK7+ y CK20- ${ }^{11}$. Una expresión CK7-/CK20 + tiene un valor predictivo positivo del $93 \%$ para metástasis de cáncer colorrectal ${ }^{14}$. Por otro lado, la proteína SATB2 es un marcador nuclear del tracto gastrointestinal inferior que aparece en el $85 \%$ de los casos de CCR, de modo que la positividad para SATB2 o CK20 diagnostica el $97 \%$ de los CCR. El marcador CDX2, aunque no es específico del CCR, pues también se expresa en otras neoplasias, refuerza el diagnóstico (Fig. 3$)^{15}$.

En las metástasis hepáticas de CCR, el tratamiento de elección es la resección quirúrgica completa, pudiendo realizar resecciones económicas siempre que se consigan márgenes libres (R0). Entendemos la resección $\mathrm{R} 0$ como aquella en la que se demuestra una ausencia total de tumor en los márgenes de resección con una distancia libre de tumor $\geq 1 \mathrm{~mm}^{16}$.
Sin embargo, cuando hay afectación biliar, la estrategia quirúrgica más aceptada es la realización de una hepatectomía anatómica reglada, que facilita la obtención de unos márgenes libres a nivel de los conductos biliares, mientras que las resecciones más económicas aumentan el riesgo de tener márgenes muy próximos o positivos a nivel biliar que aumentan el riesgo de recidiva local ${ }^{11}$. En nuestra paciente, en 2014 ya se había llevado a cabo una hepatectomía izquierda reglada, que es el tratamiento de elección para las metástasis hepáticas con crecimiento biliar, y a pesar de ello presentó una recidiva en el conducto hepático derecho. En este caso se realizó una resección de la vía biliar y del lóbulo caudado, con reconstrucción mediante hepaticoyeyunostomía en $Y$ de Roux, así como linfadenectomía, pues la sospecha inicial era un colangiocarcinoma.

Existe algún caso descrito en la literatura en que se realizó una duodenopancreatectomía cefálica por afectación del colédoco distal14.

Es importante tener un buen estudio radiológico prequirúrgico, así como un adecuado conocimiento de los distintos posibles patrones de presentación de esta condición, para planificar la estrategia quirúrgica más adecuada, pues la no sospecha o el desconocimiento de esta situación pueden llevar a realizar resecciones $\mathrm{R} 1$ con un mayor riesgo de recidiva local $^{10}$. Entendemos como resección R1 aquella en la que se demuestra la presencia microscópica de tumor en los 
márgenes de resección con una distancia libre de tumor $<1 \mathrm{~mm}^{16}$.

Según el estudio llevado a cabo por Reijonen, et al. ${ }^{4}$, las recidivas hepáticas en estos pacientes son más frecuentes $(p=0.01)$ y la supervivencia libre de recidiva hepática es menor $(p=0.031)$. Sin embargo, la supervivencia libre de enfermedad y la supervivencia global no parecen verse afectadas $(p=0.56 \text { y } p=0.831 \text {, respectivamente })^{4}$. Esto se ha relacionado con histologías menos agresivas, pues se trata en su mayoría de tumores mejor diferenciados, sin invasión venosa y con un intervalo libre de enfermedad tras la colectomía relativamente $\operatorname{largo} 0^{17}$. Los resultados de Okano, et al. ${ }^{9}$ van en consonancia con los de Reijonen, et al. ${ }^{4}$, con una supervivencia a 5 años del $80 \%$ en pacientes con afectación biliar macroscópica en comparación con el $57 \%$ en pacientes sin afectación biliar. Sin embargo, los resultados de Estrella, et al. ${ }^{7}$ no muestran diferencias en cuanto a la supervivencia global a 5 años ( $p=0.94$ ), en concordancia con otras publicaciones en las que, mediante un análisis multivariante, se descarta la influencia de la invasión biliar sobre el pronóstico ${ }^{18,19}$.

Se ha observado una asociación entre la presencia de invasión biliar y de invasión perineural, aunque tampoco parece tener valor pronóstico ${ }^{19}$. Sin embargo, la invasión linfática intrahepática, también presente en nuestra paciente, sí parece afectar a la supervivencia global ${ }^{18}$.

Los pacientes con metástasis hepáticas de CCR son tratados mediante quimioterapia (QT) neoadyuvante, y si las metástasis son resecables, son sometidos posteriormente a intervención quirúrgica. En nuestra paciente no se administró QT neoadyuvante porque la sospecha inicial era de colangiocarcinoma y no de metástasis hepáticas de CCR. No obstante, no existe suficiente evidencia de que la QT adyuvante mejore el pronóstico de los pacientes $^{14}$. En algunos estudios, como en el de Reijonen, et al. ${ }^{4}$, todos los pacientes fueron tratados con quimioterapia neoadyuvante y adyuvante, mientras que otros grupos optan por no administrar QT adyuvante ${ }^{14}$. En nuestra paciente no se ha administrado QT adyuvante.

En nuestra paciente, a pesar de que el seguimiento tras la intervención de la recidiva en el conducto hepático derecho es de apenas de unos meses, el periodo libre de enfermedad desde la hepatectomía izquierda es de más de 5 años.

\section{Conclusiones}

Ante una imagen radiológica de dilatación o crecimiento biliar, en un paciente con antecedente de CCR, se debe sospechar metástasis aunque no se observe una lesión en el parénquima hepático. Un buen estudio radiológico y la sospecha preoperatoria son fundamentales para plantear la estrategia quirúrgica, pues las resecciones deben ser anatómicas incluyendo también la resección de la vía biliar extrahepática si es preciso, con el objetivo de disminuir la probabilidad de recidiva hepática.

\section{Responsabilidades éticas}

Protección de personas y animales. Los autores declaran que para esta investigación no se han realizado experimentos en seres humanos ni en animales.

Confidencialidad de los datos. Los autores declaran que han seguido los protocolos de su centro de trabajo sobre la publicación de datos de pacientes.

Derecho a la privacidad y consentimiento informado. Los autores han obtenido el consentimiento informado de los pacientes y/o sujetos referidos en el artículo. Este documento obra en poder del autor de correspondencia.

\section{Financiamiento}

No se ha contado con ningún financiamiento.

\section{Conflicto de intereses}

Los autores declaran no tener ningún conflicto de intereses en relación con este artículo.

\section{Bibliografía}

1. Galcerán J, Ameijide A, Carulla M, Mateos A, Quirós JR, Rojas D, et al. Cancer incidence in Spain, 2015. Clin Transl Oncol. 2017;19:799-825.

2. Pawlik TM, Schulick RD, Choti MA. Expanding criteria for resectability of colorectal liver metastases. Oncologist. 2008;13:51-64.

3. De Haas RJ, Wicherts DA, Andreani P, Pascal G, Saliba F, Ichai P, et al. Impact of expanding criteria for resectability of colorectal metastases on short- and long-term outcomes after hepatic resection. Ann Surg. 2011;253:1069-79.

4. Reijonen P, Österlund P, Isoniemi H, Arola J, Nordin A. Histologically verified biliary invasion was associated with impaired liver recurrence-free survival in resected colorectal cancer liver metastases. Scand J Surg. 2018;108:201-9.

5. Nakamura T, Mitomi H, Kanazawa H, Onkura $\mathrm{Y}$, Watanabe M. Tumor budding as an index to identify high-risk patients with stage II colon cancer. Dis Colon Rectum. 2008;51:568-72.

6. Petrelli F, Pezzica E, Cabiddu M, Coinu A, Borgonovo K, Ghilardi M, et al. Tumour budding and survival in stage II colorectal cancer: a systematic review and pooled analysis. J Gastrointest Cancer. 2015; 46:212-8. 
7. Estrella JS, Othman ML, Taggart MW, Hamilton SR, Curley SA, Rashid A, et al. Intrabiliary growth of liver metastases: clinicopathologic features, prevalence, and outcome. Am J Surg Pathol. 2013;37:1571-9.

8. Kubo M, Sakamoto M, Fukushima N, Yachida S, Nakanishi Y, Shimoda T, et al. Less aggressive features of colorectal cancer with liver metastases showing macroscopic intrabiliary extension. Pathol Int. 2002;52:514-8.

9. Okano K, Yamamoto J, Moriya Y, Akasu T, Kosuge T, Sakamoto M, et al. Macroscopic intrabiliary growth of liver metastases from colorectal cancer. Surgery. 1999;126:829-34

10. Peungjesada S, Aloia TA, Kaur H, Marcal L, Choi H, Vauthey J-N, et al. Intrabiliary growth of colorectal liver metastasis: spectrum of imaging findings and implications for surgical management. Am J Roentgenol. 2013;201:W582-9.

11. Latorre Fragua RA, Manuel Vázquez A, Rodrigues Figueira Y, Ramiro Pérez C, López Marcano AJ, de la Plaza Llamas R, et al. Intrabiliary metastases in colorectal cancer: a systematic review. J Hepatobiliary Pancreat Sci. 2019;26:270-80.

12. Coppola S, Zucchini N, Romano F, Bovo G, Gilardoni E, Nespoli L, et al. Colorectal liver metastasis with intrabiliary growth: case report and review of the literature. Int J Surg Pathol. 2013;22:272-9.

13. Conci S, De Bellis M, Ruzzenente A, Capelli P, D'Onofrio M, lacono C et al. Totally intrabiliary colorectal liver metastasis mimicking intraductal growth-type cholangiocarcinoma. Updates Surg. 2016;68:211-2.
14. Nakagawa Y, Maeda A, Seita K, Kaneoka Y. Lower bile duct metastasis from rectal cancer after surgery for liver metastasis and intrahepatic bile duct metastasis: a case report. BMC Surg. 2020;20:137.

15. Magnusson K, de Wit M, Brennan DJ, Johnson LB, McGee SF, Lundberg $\mathrm{E}$, et al. SATB2 in combination with cytokeratin 20 identifies over $95 \%$ of all colorectal carcinomas. Am J Surg Pathol. 2011; 35:937-48.

16. de Haas RJ, Wicherts DA, Flores E, Azoulay D, Castaing D, Adam R. R1 resection by necessity for colorectal liver metastases: is it still a contraindication to surgery? Ann Surg. 2008;248:626-37.

17. López Gordo S, Ramos Rubio E, Torras Torra J, Lladó Garriga L, Rafecas Renau A. Crecimiento endobiliar de las metástasis de carcinoma colorrectal. Cir Esp. 2016;94:115-6.

18. de Ridder JAM, Knijn N, Wiering B, de Wilt JHW, Nagtegaal ID. Lymphatic invasion is an independent adverse prognostic factor in patients with colorectal liver metastasis. Ann Surg Oncol. 2015;22 (Suppl 3):S638-45.

19. Stift J, Graf A, Schwarz C, Tamandl D, Starlinger P, Herac M, et al. Microscopic biliary and perineural invasion and clinical outcome after neoadjuvant bevacizumab-based chemotherapy and liver resection in patients with colorectal liver metastases. Eur J Surg Oncol. 2018; 44:139-47. 